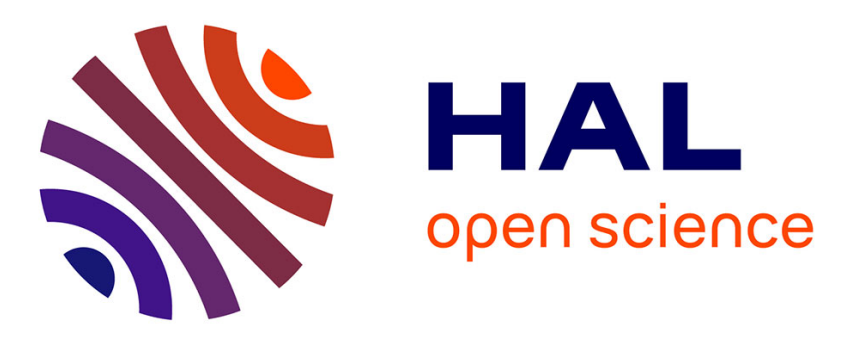

\title{
A Physical Model for Wildland Fires
}

Jacques Henri Balbi, Frédéric Morandini, Xavier Silvani, Jean Baptiste Filippi, Frédéric Rinieri

\section{To cite this version:}

Jacques Henri Balbi, Frédéric Morandini, Xavier Silvani, Jean Baptiste Filippi, Frédéric Rinieri. A Physical Model for Wildland Fires. Combustion and Flame, 2009, 156 (12), pp.2217-2230. 10.1016/j.combustflame.2009.07.010 . hal-00593608

\section{HAL Id: hal-00593608 https://hal.science/hal-00593608}

Submitted on 16 May 2011

HAL is a multi-disciplinary open access archive for the deposit and dissemination of scientific research documents, whether they are published or not. The documents may come from teaching and research institutions in France or abroad, or from public or private research centers.
L'archive ouverte pluridisciplinaire HAL, est destinée au dépôt et à la diffusion de documents scientifiques de niveau recherche, publiés ou non, émanant des établissements d'enseignement et de recherche français ou étrangers, des laboratoires publics ou privés. 


\section{A Physical Model for Wildland Fires}

J.H. Balbi*, J.B. Filippi, F. Morandini, F. Rinieri and X. Silvani

Corresponding author: Pr. Jacques-Henri Balbi, balbi@univ-corse.fr.

Laboratory for the Physical Systems of the Environment CNRS UMR 6134 / University of Corsica

Quartier Grossetti

20250 CORTE

Under consideration for publication in

Combustion and Flame 


\begin{abstract}
This work presents an enhanced version of a simple model of surface fire spread, already reported in [1]. The simplicity of the original model was preserved and the 'faster than real time' simulation speed were retained. The empirical relations were replaced by physical equations using flame geometry considerations, and it now uses only two model parameters. This model was tested and validated using a large set of lab and field scale experiments, carried out across fuel beds under different slope and wind conditions. Finally, this model was implemented as the physical core of a wild fire simulation tool for field scale fires and the case of a large wild fire was successfully treated as a first code evaluation.
\end{abstract}

Keywords: wildland fire, physical model, fire experiments 


\section{Nomenclature}

a moisture factor

A energy ratio between incident radiant energy and ignition energy of wet fuel

$\mathrm{A}_{0} \quad$ energy ratio between incident radiant energy and ignition energy of dry fuel

B Stephan-Boltzman constant

$\mathrm{W} \cdot \mathrm{m}^{-2} \mathrm{~K}^{-1}$

Cp gas calorific capacity

$\mathrm{J} . \mathrm{kg}^{-1 \circ} \mathrm{K}^{-1}$

$\mathrm{Cp}_{\mathrm{v}} \quad$ vegetal calorific capacity

J.kg ${ }^{-1} \mathrm{~K}^{-1}$

e thickness of the vegetal stratum

$\mathrm{m}$

$g$ gravitation

$\mathrm{m} \cdot \mathrm{s}^{-2}$

$\mathrm{H} \quad$ flame height

$\mathrm{m}$

$\ell \quad$ flame length

$\mathrm{m}$

L flame depth

$\mathrm{m}$

m moisture content

$\%$

$\vec{N} \quad$ unit normal vector to the ground

$\vec{n} \quad$ unit normal vector to the front

$\vec{p} \quad$ unit slope vector

Q power of the fire heat release

$\mathrm{W} . \mathrm{m}^{-1}$

$\vec{R}=R \vec{n}$ ROS vector

R ROS

$\mathrm{m} \cdot \mathrm{s}^{-1}$

$\mathrm{R}_{0} \quad$ ROS for no-slope, no wind

$\mathrm{m} . \mathrm{s}^{-1}$

$r=\frac{R}{R_{O}}$ reduced ROS

$\mathrm{R}_{00}$ energy ratio between incident radiant energy emitted from the flame base and ignition energy of dry fuel $\mathrm{kg} \cdot \mathrm{m}^{-2} \cdot \mathrm{s}^{-1}$

$\mathrm{v}_{0} \quad$ ROS coefficient

$\mathrm{m} \cdot \mathrm{s}^{-1}$ 
$T \quad$ flame gas temperature

Ta air temperature

$\mathrm{Ti}$ ignition temperature

$T v \quad$ vegetal fuel temperature

$\vec{U} \quad$ wind velocity vector

U wind velocity

$\vec{u}=u \vec{z}$ buoyancy velocity vector

$u \quad$ buoyancy velocity

$\mathrm{m} \cdot \mathrm{s}^{-1}$

$u_{0} \quad$ buoyancy velocity component for a zero slope

$\mathrm{m} . \mathrm{s}^{-1}$

$u_{00} \quad$ coefficient of vertical velocity

$\mathrm{m}^{3} \cdot \mathrm{kg}^{-1}$

$\mathrm{x}$ distance to the flame

$\mathrm{m}$

$\vec{z} \quad$ unit vertical vector

Greek symbols
$\alpha \quad$ slope angle
$\beta \quad$ packing ratio

$\Delta \mathrm{H} \quad$ combustion enthalpy

J.kg ${ }^{-1}$

$\Delta \mathrm{h}_{\mathrm{v}} \quad$ moisture evaporation enthalpy

$\mathrm{J} . \mathrm{kg}^{-1}$

$\phi \quad$ angle between $\vec{p}$ and $\vec{n}$

$\phi_{\mathrm{B}} \quad$ flux radiated by the flame base

W. $\mathrm{m}^{-2}$

$\phi_{\mathrm{F}} \quad$ flux radiated by the flame

W. $\mathrm{m}^{-2}$

$\psi \quad$ angle between $\vec{U}$ and $\vec{n}$

flame emissivity 
$\gamma \quad$ tilt angle between $\vec{N}$ and the flame

$v$ absorption coefficient for the thermal radiation

$\rho \quad$ gas flame density

kg. $\mathrm{m}^{-3}$

$\rho_{\mathrm{a}} \quad$ surrounding air density

$\mathrm{kg} \cdot \mathrm{m}^{-3}$

$\sigma \quad$ surface density of vegetal fuel

$\mathrm{kg} \cdot \mathrm{m}^{-2}$

$\dot{\sigma} \quad$ vegetal mass loss rate

$\mathrm{kg} \cdot \mathrm{m}^{-2} \cdot \mathrm{s}^{-1}$

$\sigma_{\mathrm{H}_{2} \mathrm{O}}$ surface density of vegetal moisture content

kg. $\mathrm{m}^{-2}$

$\tau \quad$ residence time

S

$\theta \quad$ view angle of the flame 


\section{1 - Introduction}

Wildfires are responsible for several types of devastating damage, including a loss of biodiversity, decrease in forests, alteration of the landscape, soil degradation, increase in the greenhouse effect, etc. To combat this natural hazard, for several decades the scientific community has tried to predict the propagation of forest fires, with the goal of supporting fire fighting and fire prevention decisions. With this in mind, several strategies for forest fire modelling have been developed. For fire prevention, these models must allow the dimensioning of fuel breaks and management of the vegetal cover: the relevancy of the simulated results is of the first order of importance before any consideration about the computational cost and memory use. For assist with the decision making process during fire fighting, the CPU time cost of the model must be shorter than the real time scale of the fire spread in order to adjust the fire fighting operations based on the predicted fire behaviour. The computational cost and memory use must be low in order to be efficient during a field scale fire. In either case - fire prevention or fire fighting - the forest managers and fire fighters want to know the kinematics of the fire front and the related characteristics (heat release, flame height and fire front depth, flame angle, temperature and radiant heat fluxes). Numerous modelling approaches for wildfire propagation have been developed over the years. These can be sorted according to the following classes.

-Empirical models (see [2] and [3]):

An algebraic law defines the rate of fire spread R (ROS) as a function of the wind flow velocity $\mathrm{U}$, the slope ground $\alpha$, the moisture content $\mathrm{m}$ and some parameters called $f$ that characterise the vegetal fuel. This law is derived according to a more or less arbitrary method:

$$
R=R(U, \alpha, m, f)
$$


The parameters introduced in this relation are set up using some experimental data in order to minimize the gap between the modelled and measured ROS. This approach is simple and computationally efficient but the model is only valid in the range of experiments for which it was validated. Peculiarly, the change from lab to field scale experiments is not supported, but involves a new calibration of the parameters.

-Semi-empirical models [4] and [5]:

These involve at least a physical conservation law, i.e. the thermal budget for the vegetal fuel, which is derived and closed using some empirical sub-models. These semi-empirical models present the same characteristics of simplicity and computational efficiency as fully empirical models. However, they also have numerous parameters to calibrate and an operating range that is limited to the validation framework. Their main advantage over the previous models concerns their greater ability to be converted from one scale to another because they depict some relevant aspects of fire physics. The most famous is the Rothermel Model [4], which is implemented in full scale simulation code as BEHAVE [12] and FARSITE [13].

-Reduced physical models [6], [7] and [8]

In this strategy, the physical laws are more fully taken into account. Specifically, the thermal budget in the solid fuel is described using a partial differential equation for a reactiondiffusion process. The computational cost increases because the solid surface covered by the fuel must be discretised with a convenient mesh and this can lead to real time overlap.

-Full-physical models [9], [10] and [11]

This approach is based on the numerical resolution of the conservation laws for mass, momentum and energy in a multiphase medium formed by the vegetal fuel and the surrounding air flow. These laws lead to a system of coupled non-linear partial differential equations (PDE), the numerical resolution of which needs a 3D mesh grid. The large range of 
the time and space scales involved in this approach yields high computational costs in term of CPU time and memory storage. The execution time for each simulation is far from real time.

Furthermore, despite its original ambition, this approach is not fully physical. Indeed, source terms and unclosed terms in the PDE system invoke semi-empirical models with numerous parameters to fit.

In the end, reduced or full-physical models are reliable for providing approximations of the 3D Eulerian fields proceeding from the fire spread as velocity, mass or mixture fraction, and temperature. They account for the academic knowledge acquired about natural fires but, up to now, it has not been possible to use them for fire fighting or forest management. The computational costs of such numerical tools are still too great to use them in real fire cases. Furthermore, the setup of the sub-model parameters is prohibitive in such cases.

Empirical models can be useful for computing the ROS for fires in the field but cannot provide any physical quantity associated with the fire spread (heat flux, gas or fuel temperature, fire size...). Semi empirical models are therefore the best candidates for operational use and are extensively used in this way. Nevertheless, they do not conveniently introduce the influences of open areas (ground slope, wind flow, fuel features...) and do not provide good support for the geometrical and thermodynamic characteristics of the flame front. The departure from real cases can be extremely strong in cases for which they have not been tested. Finally, they also need numerous parameters to set-up.

These are reasons why we purpose a new kind of model:

-A simplified full-physical model.

This model is based on a simplified modelling of the governing transport phenomena from the fire: in this sense, it is a full-physical model because, beyond the ROS, it provides every global physical quantity related to the fire front. Its CPU execution time must be very low to allow it to be used in the real time conditions for a real wildland fire. It must only use a few 
geometrical points for initiating the fire front propagation and must be valid for every fire scale. Its accuracy must be reasonably good in every case.

\section{2 - A simplified physical model}

\section{2-a. Scientific objectives}

We can now review the key points of the model that forms the basis for the one described in the present paper [1] (Balbi et al., 2007). Using some appropriate assumptions, the usual budget equations yield a system of two coupled non-linear algebraic equations relating the rate of spread (ROS) and the flame angle. So, the computational time is shorter than the real time. The resolution just depends on a set of four parameters, initially set-up through an optimization procedure, whereas the set of thermal and geometrical variables are computed by the model. The original paper [1] showed that this first simplified model was reliable when used in a set of lab-scale and field scale experiments.

The objectives described in the previous section - mainly the reliability of a physical real time simulation - were reached. The aim of the present paper is to propose a new version that upgrades the original model in several points:

- The empirical relations in the original version of the model (MacCaffrey flame height intensity relation [14], radiant loss from the flame) have been suppressed using geometrical considerations.

- The formal development leads to a single algebraic relation, which replaces the original set of two coupled non linear equations.

- The reduced rate of spread is given by a universal formula without any parameter. Two parametric terms (rather than the four initially used) are needed for the complete closure of the system and can be computed when the vegetal cover is completely known (in the previous model, they had to be fixed using an optimization procedure). 
- This new version is implemented in a wildfire simulator.

The next section will present the assumptions from which the thermodynamic fundamentals are expressed, in a simplified form.

\section{2-b Theoretical assumptions}

The simplified model is based on the set of 10 assumptions presented below. Comments relative to these assumptions are presented in italics for the sake of simplicity.

\section{H1 - Triangular flame}

The normal cut of the flame volume is triangular (see Fig. 2).

Considering the average flame as triangular can obviously appear to be a rough approximation, but this allows its geometry to be expressed in a very simple form, consistent with observed results and minimizing the number of geometrical parameters.

H2 - Negligible heat convection ahead of the flame front Such a strong assumption proceeds from the following.

1 - The flame just above the vegetal stratum (the continuous flame region according to MacCaffrey [14]) forms a strong ascending heat flow, the inertia of which stops the upwind flow from crossing the flame. This is illustrated in Fig. 2, in which the incident stream lines are reoriented in the convective column. The consequence is that there is no heat transport due to the wind flowing through the flame front. However, the heat convection from the intermittent part of the flame cannot be neglected, but this does not affect the vegetal stratum. Therefore, under the flame, no significant heat transport due to an up-wind flow by convection should be expected. 
2 - Such a convective column of hot gases entrains the fresh oxidiser gases ahead of the flame, just as diffusion flames do. In Fig. 2, over the portion KO, the flow rate for the entrained surrounding air is weak and this air flow is warmed up by the flame. Therefore, the heat transport from this a priori cooling weak air stream can be considered as negligible in comparison with the heat transport due to the incident thermal radiation.

3 - Such an assumption has been experimentally observed to be relevant in large scale fires governed by weak wind flow: the thermal budget ahead of a flame front is essentially radiative (see Fig. 3 courtesy of Silvani and Morandini [15]).

4 - Recent studies of the stream lines under flames spreading over a vegetal fuel using Particule Intercorrelation Velocimetry [16] have been performed at the lab scale. Air flow over the fuel bed above the flame is negligible.

Finally, the convective heat transport above the flame can be viewed as negligible in the major parts of natural spreading fires.

Such an assumption remains valid for a 'plume dominated fire' or a 'wind driven fire', i.e. for weak or strong wind flows. The results presented in the following (Fig. 16) will illustrate that a model based on the assumption of no significant convective heat transport ahead of the flame front leads to relevant results even in the case of a strong tilt angle (up to $70^{\circ}$ ).

Nevertheless, such an assumption is no longer valid when the flame angle is close to vertical (about $90^{\circ}$ ). The heat transport regime then becomes purely convective.

H3 - Velocity composition and tilt flame angle

The velocity $\vec{V}$ into the flame results from the sum of the incident wind velocity $\vec{U}$ and the natural convective velocity into the flame $\vec{u}$ (due to the heat release). The flame is tilted toward the soil in the direction of the normal component of this velocity field $\vec{V}=\vec{U}+\vec{u}$. 
The velocity composition assumption allows a single definition for the flame tilt angle in every configuration, particularly when the main slope direction and wind velocity are not aligned.

H4 - State equation

The gases are considered to perfect ones and the thermodynamic transformations are isobaric (because of the validity of the Low Mach Number approximation in natural fires). We also assumed the existence of an average flame temperature.

The assumption of a perfect gas mixture is usual in fire safety science and so is not commented on here.

H5 - 'Stoechiometric' inflow

The air entrained into the flame by natural convection allows the complete oxidation of reactive gases, as the equivalent chemical reaction was governed by a 'stoechiometric' mixture between combustible and (air) oxidizer gases.

For chemical processes, the equivalent reaction is usually considered to be:

$$
\mathrm{C}+\mathrm{O}_{2} \rightarrow \mathrm{CO}_{2}
$$

This consists of a strong reduction in the chemical processes, but is realistic. Based on our present understanding, the chemical analysis of any vegetal fuel shows that it is mainly composed of carbon and oxygen (about 40\% of the vegetal fuel mass [17]). Such considerations yield a 'stoechiometric' coefficient $s=9$, i.e. for the complete burning of $1 \mathrm{~kg}$ of gas pyrolisis, $9 \mathrm{~kg}$ of air are needed.

H6 - A surface fuel distribution

The vegetal cover is distributed homogeneously in domains; each elementary part of the domain totally burns and there is a single vegetal stratum. 
This assumption suggests that the model is defined for surface fires in which the vegetal stratum is considered be homogenous. We will nevertheless examine some cases of inhomogeneous strata and the related set-up of the model for these cases.

H7 - Constant mass loss rate

We assume that the mass loss for the vegetal fuel is linear versus time as soon as the gas temperature reaches the temperature of fuel ignition (about $300{ }^{\circ} \mathrm{C}$ in the current literature).

This conveniently imposes the model for the thermal degradation of a solid under heat fluxes, greatly simplifying the usual exponential Arrhenius model.

\section{H8 - Radiative tangent plane}

For every point close to the flame front, this one can be considered as its tangent plane of infinite length, the height of which is equal to the flame length. (see Figs. 1 and 2).

This assumption is consistent with assumption H9, implying that only the vegetal part under the flame is affected by the thermal transfer, i.e. when the distance to the flame front is weak. This reinforces the idea that the flame behaves as a radiant plane for the heated unburned fuel.

H9 - Preheating under the flame

The radiant plane heats the unburned fuel only under the flame.

In the unburned part of the vegetal cover, there is an induced air flow that cools the fuel exposed to long range radiation. Out of the flame, the two effects compensate each other. But this air stream is derived toward the convective column of hot gases and does not flow inside the flame, creating a region of a quite null velocity field just above the flame (see H2). This yields the assumption that the preheating from the fire only takes place under the flame. 
H10 - Radiative factor

The amount of energy emitted by radiation is a decreasing function of the surface-to-volume ratio of the flame.

This expresses the rare experimental data concerning the radiant factor [6] and the following scale dependence: if, by considering a constant flame surface, the volume of the flame decreases, the proportion of energy emitted by radiation to the overall released energy must also decrease.

\section{2-c Governing equations}

\section{$\underline{\text { E1 - Mass budget }}$}

Along a linear meter of fire front, the flow rate of the pyrolyse gas is given by $L \dot{\sigma}$, with $L$ standing for the fire front depth and $\dot{\sigma}$ the mass loss rate. Following hypothesis H6, the air enters the flame according the 'stoechiometric' proportion $s$, leading to a flow rate $s L \dot{\sigma}$.

The mass flow rate at the half-height of the modelled flame is: $\rho \frac{L}{2} \vec{u} \cdot \vec{N}=\rho \frac{L}{2} \cos \alpha u$, where $\alpha$ stands for the slope angle. The mass flow rate is therefore: $\rho \frac{L}{2} \cos \alpha u=L \dot{\sigma}+s L \dot{\sigma}$. This expression yields:

$$
\begin{gathered}
u=\frac{u_{0}}{\cos \alpha} \\
\text { with } u_{0}=\frac{2(1+s)}{\rho} \dot{\sigma}
\end{gathered}
$$

where $u_{0}$ stands for the ascension of the velocity of the combustion gas for a null slope ( $\rho$ and $\mathrm{T}$ being considered as constant for the flame). This velocity $u$ varies with the ground slope according to (1). 


\section{$\underline{\text { E2 - Velocity composition and flame tilt angle: }}$}

According to $\mathrm{H} 3$, the gas velocity in the flame follows: $\vec{V}=\vec{U}+\vec{u}$, and this is the normal component of the velocity field that tilts the flame (see Figs. 1 and 2):

$\vec{V}_{\text {normal }}=U \cos \psi \vec{n}+u(\cos \alpha \vec{N}+\sin \alpha \cos \phi \vec{n})$

where $\vec{n}$ is the unit vector normal to the flame; $\vec{N}$ is the unit vector normal to the plane; $\phi$ is the angle between the ground slope vector $\vec{p}$ and $\vec{n}$. Finally, $\psi$ is the angle between $\vec{n}$ and the wind $\vec{U}$.

The resulting tilt angle is given by: $\tan \gamma=\frac{u \sin \alpha \cos \phi+U \cos \psi}{u \cos \alpha}$.

$$
\tan \gamma=\tan \alpha \cos \phi+\frac{U}{u_{0}} \cos \psi
$$

Then, the tilt angle depends on the wind $\mathrm{U}$ and the slope $\alpha$, but also on the front normal $\vec{n}$.

\section{E3 - Thermal balance:}

The thermal budget in the flame (for a linear meter of fire front) indicates that the heat released each second by the gaseous combustion $Q$ leads to a radiated part $\chi Q$.

$\rho C p u \frac{L}{2} T \cos \alpha-(s+1) L C p \dot{\sigma} T_{a}=(1-\chi) Q$

where $T$ is the mean temperature of the flame and $T_{a}$ is the ambient temperature; $C_{p}$ stands for the calorific capacity.

According to Eq. (1), $\rho u \frac{L}{2} \cos \alpha=(1+s) L \dot{\sigma}$ and $Q=\Delta H L \dot{\sigma}$ with $\Delta H$ standing for the combustion enthalpy. This yields a definition for the flame averaged temperature.

$$
T=T a+(1-\chi) \frac{\Delta H}{(1+s) C_{p}}
$$

E4 - Flame height: 
The equation for the vertical momentum applied to the flame gives:

$$
\rho \frac{d u}{d t}=\left(\rho_{a}-\rho\right) g
$$

i.e. $\frac{d u}{d t}=g^{*}$ with $g^{*}=g\left(\frac{T}{T_{a}}-1\right)$. This leads us to consider a uniformly accelerated motion of hot gases yielding to the vertical velocity at the mid-height flame to be

$$
\begin{gathered}
u ?=2 g^{*} \frac{H}{2} \\
\text { with } H=\frac{u^{2}}{g^{*}} \\
H=\frac{u_{0}^{2}}{g^{*} \cos ? \alpha} .
\end{gathered}
$$

And following Eq. (1),

\section{$\underline{\text { E5 - Radiation sub-model: }}$}

A vegetal cell receives thermal radiation $\phi_{\mathrm{F}}$ from the flame region above the vegetation and radiation $\phi_{\mathrm{B}}$ in the flame part (and chars) inside the vegetation.

This second term is considered to be a 'grey' radiant panel of infinite length and of height $e$. Its temperature is $\mathrm{T}$ and its emissivity is $\varepsilon_{\mathrm{B}}$. The corresponding radiant intensity is given by $\varepsilon_{\mathrm{B}} B$ $T^{4}$ along the $\vec{x}$ axis (B is the Stefan-Boltzmann constant). This impacting thermal radiation uniformly decreases over the vegetal fuel and is damped at the optical length $\delta$.

At the distance $\mathrm{x}$ from the fire front, the heat flux $\phi_{\mathrm{B}}$ is given according to:

$\phi_{B}=\varepsilon_{B} B T^{4}\left(1-\frac{x}{\delta}\right) \frac{e}{\delta}$ if $\mathrm{x}<\delta$ and $\phi_{B}=0$ if $\mathrm{x}>\delta$

The flame region above the vegetation is also considered as an infinite grey panel of length $\ell$, temperature $\mathrm{T}$ and emissivity $\varepsilon$. Furthermore, the radiant heat flux from the burning vegetal fuel is given by: 
$\phi_{F}=\varepsilon B T^{4} \frac{1}{\pi} \int \frac{\cos \varphi_{1} \cos \varphi_{2}}{R ?} d S$ where $\mathrm{R}$ is the distance between one point of the panel and the vegetal cell. After a longitudinal integration, this expression becomes:

$$
\phi_{F}=\frac{\varepsilon}{2} B T^{4}(1-\cos \theta)
$$

where $\theta$ is the view angle.

E6 - Pre-heating sub-model:

The thermal budget in a vegetal cell below the flame can be written as:

$$
\sigma C_{p} \frac{d T_{v}}{d t}=\phi_{B}+v \phi_{F}-\Delta h_{v} \frac{d \sigma_{H_{2} O}}{d t}
$$

where $\mathrm{TV}$ is the mean temperature of the cell, $\Delta \mathrm{h}_{\mathrm{v}}$ is the evaporation enthalpy and $\sigma_{\mathrm{H} 2 \mathrm{O}}$

represents the water load.

According to $\mathrm{H} 2$, no convection occurs below the flame and the air velocity is zero. The fraction of the flame radiation absorbed by the fuel is denoted as $v$ and given by the expression:

$$
v=\inf \left(\frac{e}{\delta} ; 1\right)
$$

where $e$ is the thickness of the strata and $\delta$ the optical length through the vegetal given by: $\delta=4 / \mathrm{s}_{\mathrm{v}} \beta ; \mathrm{s}_{\mathrm{v}}$ is the surface to volume ratio and $\beta$ is the packing ratio.

As a consequence, we use the space variable, following the normal $\vec{n}(\mathrm{dx}=-\mathrm{R} \mathrm{dt})$, and integrate over the interval $[0 ; \sup (\delta: \ell \sin \gamma)]$ with $\ell$ as the flame length. Indeed, beyond the segment $[0 ; \sup (\delta: \ell \sin \gamma)]$, the radiation and the air cooling by convection compensate one another according to H9:

$$
R \sigma\left[C_{p}\left(T i-T_{a}\right)+m \Delta h_{v}\right]=\int_{0}^{\delta} \phi_{B} d x+v \int_{0}^{\ell \sin \gamma} \phi_{F} d x
$$

yielding to 


$$
\begin{gathered}
\int_{0}^{\delta} \phi_{B} d x=\frac{\varepsilon_{B}}{2} B T^{4} e \\
\int_{0}^{\ell \sin \gamma} \phi_{F} d x=\frac{\varepsilon}{2} B T^{4} \ell(1+\sin \gamma-\cos \gamma)
\end{gathered}
$$

\section{$\underline{\text { E7 - Radiant fraction }}$}

According to H10, the radiant fraction $\chi$ decreases with an increase in the volume/surface ratio V/S of the flame, which is expressed by: $\chi=\frac{\chi_{0}}{1+\mu \frac{V}{S}}$

where $\chi_{0}$ is the radiative fraction when $\frac{V}{S} \rightarrow 0$, according to the assumption of a thin flame, and,

$V=0.5 \bar{H} \cdot L \cdot 1 \quad$ (see Fig. 2)

$S=\ell .1$

$\frac{V}{S}=0.5 L \cos \gamma$. Nevertheless, $L=R \tau$, with $\tau$ the residence time and $v_{0}=2 \tau \mu$, with the dimension of a velocity, is proportional to the ROS for high values of $\gamma$.

$$
\chi=\frac{\chi_{0}}{1+\frac{R}{v_{0}} \cos \gamma}
$$

MacCaffrey's correlation [18] indicates that the radiant fraction is constant as long as the flame remains relatively thin, and then decreases slowly with an increasing flame thickness.

The thin flame coincides with the case of a slow fire spread: with $\gamma=0, \mathrm{R}=\mathrm{R}_{0}, \chi=\frac{\chi_{0}}{1+\frac{R_{0}}{v_{0}}}$.

If $\mathrm{R}_{0}$ varies (with $\sigma$, e or $\mathrm{m}$ ), $\chi$ must remain constant. Then $\mathrm{v}_{0}=\mathrm{nR}_{0}$ where the number $\mathrm{n}$ must be great enough to ensure a slow variation of $\chi$ with $\mathrm{R}$ (i.e. with the thickness). 
The code validations presented in the following illustrate that the fit to the value $n=12$ is convenient. The choice of this value eliminates the parameter $\mathrm{v}_{0}$ in the original model.

\section{E8 - Rate of spread (ROS):}

We can now set all sub-models the ones with the others, considering two distinct fire dynamics:

- slow backing fire spread: $\gamma \leq 0$. This is the case where $\phi_{\mathrm{F}}$ does not play a significant role, i.e. when the flame axis is normal to the ground or tilted toward the already burned vegetation. In this case, Equations (6.a) and (6.b) yield:

$$
R_{0}=\frac{\varepsilon_{B} B T^{4} e}{2 \sigma\left[C_{p}\left(T_{i}-T_{a}\right)+m \Delta h_{v}\right]} .
$$

This rate of spread is low and does not depend on the flame tilt angle; we note:

$$
R_{0}=\frac{e}{\sigma} \frac{R_{00}}{(1+a m)}
$$

with $R_{00}=\frac{\varepsilon_{B} B T^{4}}{2 C_{p}\left(T_{i}-T_{a}\right)}$ and $a=\frac{\Delta h_{v}}{C_{p}\left(T_{i}-T_{a}\right)}$.

- fast fire spread: $\gamma>0$

The flame is tilted toward the unburned vegetation: $\gamma>0$; the heat flux impinging the fuel is stronger, and therefore the rate of spread is also greater. Using Eq. (6), Eq. (6') and Eq. (6'), we obtain:

$$
R=R_{0}+\frac{v \varepsilon B T^{4} \ell}{2 \sigma\left[C_{p}\left(T_{i}-T_{a}\right)+m \Delta h_{v}\right]}(1+\sin \gamma-\cos \gamma)
$$

However, $\varepsilon B T^{4} \ell=\frac{\chi}{2} Q=\frac{\chi}{2} \Delta H \dot{\sigma} L$. According to assumption H7, we have $\dot{\sigma}=\frac{\sigma}{\tau}$ and $L=R \tau$, yielding: $\varepsilon B T^{4} \ell=0.5 \chi \Delta H \sigma R$. This results in: 


$$
\begin{gathered}
R=R_{0}+A \frac{R}{1+\frac{R}{v_{0}} \cos \gamma}(1+\sin \gamma-\cos \gamma) \\
\text { with } A=\frac{v A_{0}}{(1+a m)}, A_{0}=\frac{\chi_{0} \Delta H}{4 C_{p}\left(T_{i}-T_{a}\right)}
\end{gathered}
$$

leading to the following exact solution:

$$
\begin{aligned}
& R=0.5\left[R_{a}+\left(R_{a}{ }^{2}+\frac{4 v_{0} R_{0}}{\cos \gamma}\right)^{0.5}\right] \\
& R_{a}=R_{0}+A v_{0} \frac{1+\sin \gamma-\cos \gamma}{\cos \gamma}-\frac{v_{0}}{\cos \gamma} . \\
& \text { 2-d Exact solutions and asymptotic behaviour }
\end{aligned}
$$

The exact solution of the model Eq. (9) is given by Eq. (11) and Eq. (11'). R is an increasing function of $\gamma$.

This can be approximated for weak $\gamma$ as follows. Eq. (9) leads to: $R=R_{0}+A R_{0} \sin \gamma$ using Eq. (2) and considering a rectilinear fire front (with a weak curvature radius).

$$
R=R_{0}+A R_{0}\left[\tan \alpha+\frac{U}{v_{0}}\right]
$$

For high values of $\gamma$, Eq. (9) has an asymptotic solution: one can neglect $R_{0}$ in front of $R$. This yields:

$$
\begin{aligned}
& R=\frac{v_{0}}{\cos \gamma}[A(1+\sin \gamma-\cos \gamma)-1] \\
& R=\frac{v_{0} \sin \gamma}{\cos \gamma}\left[\frac{A(1+\sin \gamma)-1}{\sin \gamma}\right]-v_{0} A
\end{aligned}
$$

and for a sufficiently high $\gamma$, Eq. (9) obeys the asymptote:

$$
R=v_{0}\left(\tan \alpha+\frac{U}{u_{0}}\right)(2 A-1)-v_{0} A
$$


Finally, the law for R exhibits a linear dependence on $U$ for strong slope angles, whereas for weaker values of $\gamma$, the relation between the ROS and wind velocity is strongly non-linear.

Figure 4 shows the plot of two asymptotes given by Eq. (12) and Eq. (12'), with the curve plotting the complete solution given in Eq. (9). Two cases must be considered:

- weak slopes: $\tan \alpha<\frac{A}{2 A-1}$.

A is often about $1-1.5$, implying $\alpha<37^{\circ}$ : this is shown in Fig. 4.1. In this graph, at the beginning, the dependence of $r$ on $U$ is linearly increasing at a constant rate $\mathrm{p}_{0}=\mathrm{A}$. This rate increases until it reaches the asymptote governed by the coefficient $\mathrm{p}_{\infty}=\frac{v_{0}}{R_{0}}(2 A-1)$. Keeping the value $A=1.5$ and with $\mathrm{v}_{0}=12 \mathrm{R}_{0}$, yields $\mathrm{p}_{0}=1.5$ and $\mathrm{p}_{\infty}=24$.

Then, the ROS dependence on $U$ is locally linear for small values and large values of $U$, but far from these two extrema, the dependence is non-linear.

- $\quad$ strong slope: $\tan \alpha>\frac{A}{2 A-1}$

The ROS presents the same asymptotic behaviour for weak and strong wind flows (Fig. 4.2), but in the second case, the ROS increases more strongly from the first asymptote to the next one. In both cases, it can be observed that the behaviour is fully non-linear. However, it becomes linear for strong wind velocities, although the fire spread remains governed by the pure thermal radiation $(\mathrm{H} 2)$.

\section{2-e Parameters}

This section discusses the models parameters. The model uses a set of four parameters: $\mathrm{R}_{0}, \mathrm{u}_{0}$, A and $v_{0}$. We recall that, for a homogeneous vegetative stratum, the last one is given by: $v_{0}=$ $12 \mathrm{R}_{0}$ 
The three others involve input variables for the model characterizing the vegetal stratum (moisture content, fuel load) and also, others coefficients varying a few between different vegetal fuels.

$\mathrm{R}_{0}$, defined by Eqs. (8) and (8'), depends on $(e, \sigma, m)$, which characterize the vegetal stratum, along with $\mathrm{R}_{00}$ and a. For example, the coefficient a can be estimated:

- by using its algebraic expression ( $\left.8^{\prime}\right)$, because the three quantities from which a is formed $\left(\Delta \mathrm{h}_{\mathrm{v}}, \mathrm{Cp}, \mathrm{T}_{\mathrm{i}}-\mathrm{T}_{\mathrm{a}}\right)$ are a priori known and do not vary significantly from one vegetal species to another.

- or by using the numerous experimental results available in the literature. These two approaches provide values that are close the each other. From now on, we will use the universal coefficient: $\mathrm{a}=0.05$.

$\mathrm{R}_{00}$ is a priori 'less' universal because it depends on the quantity $\mathrm{T}^{4}$, and $\mathrm{T}$ changes slightly from one type of vegetation to another, according to the external conditions. An evaluation of $R_{00}$ for a bed of pine needles can be given as: $R_{00}=0.05 \mathrm{~kg} \cdot \mathrm{m}^{-2} \mathrm{~s}^{-1}$.

Because of this dependence, a fitting of $\mathrm{R}_{0}\left(\right.$ or $\left.\mathrm{R}_{00}\right)$ in every case is preferred, considering that the rate of spread on flat ground without wind is easily measurable. However, Eq. (8') will be useful from a qualitative point of view.

The parameter $\mathrm{u}_{0}$ is given through Eq. (1'), and according to $\mathrm{H} 7: \dot{\sigma}=\sigma / \tau$ where: $u_{0}=u_{00} \sigma / \tau$ with $u_{00}=2(s+1) / \rho$. We already considered that $\mathrm{s}=9$, and according to H4: $\rho=\rho a \frac{T a}{T}$ where $\mathrm{T}$ varies slightly from one type of vegetation to another $(\mathrm{T} \sim 1100 \mathrm{~K})$. This leads to: $u_{00}=80 \mathrm{~kg}^{-1} \cdot \mathrm{m}^{3}$. This value has been confirmed in experimental cases (for example, in [19] with $\sigma=0.5 \mathrm{~kg} . \mathrm{m}^{-2} ; \tau=20 \mathrm{~s} ; u_{0}=2 \mathrm{~m} . \mathrm{s}^{-1}$ ). Here again, care must be taken when fitting this value from experimental data and using its algebraic expression for qualitative considerations. 
Finally, A is provided by (10) and (6"') is expressed using the universal coefficient $A_{0}$, which can be computed with an analytical expression where several terms slowly vary from one species to another.

$A_{0}=\frac{\chi_{0} \Delta H}{4 C p\left(T i_{-} T a\right)}$ where $\chi_{0}=0.3 ; \Delta \mathrm{H}=1.8 .10^{7} \mathrm{~J}_{\mathrm{kg}}{ }^{-1} ; \mathrm{Cp}_{\mathrm{v}}=2.10^{3} \mathrm{~J}_{\mathrm{kg}} \mathrm{kg}^{-1}$ leads to

$\mathrm{A}_{0}=2.25$. This value has been confirmed by numerical evaluations of this coefficient in experiments (see [19], as above).

\section{2-f Synthesis of the model}

Our model gives the radiant flux (5), the flame temperature (3), the flame height (4), the flame depth, $\mathrm{L}=\mathrm{R} \tau$, the heat released by time unit, $Q=\Delta H \sigma R$,

the tilt angle, $\tan \gamma=\tan \alpha \cos \phi+\frac{U}{u_{0}} \cos \psi$

and the ROS:

$$
\begin{aligned}
& \text { if } \gamma \leq 0, r=\frac{R}{R_{0}}=1 \\
& \text { if } \gamma \leq 0, r=1+\frac{\operatorname{Ar}(1+\sin \gamma-\cos \gamma)}{1+\frac{r}{12} \cos \gamma} .
\end{aligned}
$$

We have a physical model given by the algebraic relation (2)-(13); the dynamics of the fire front can therefore be simulated for any slope and wind conditions for a large range of spatial scales, with a low CPU time cost.

This model depends on 3 parameters, $\mathrm{A}, \mathrm{R}_{0}$ and $\mathrm{u}_{0}$ :

- A is always computed using $A=v \frac{A_{0}}{1+a m}$

with $\mathrm{A} 0=2.25, \mathrm{a}=0.05, v=\inf \left(\frac{e}{\delta} ; 1\right), \delta=\frac{4}{s_{v} \beta}$ and $\frac{e}{\delta}=\frac{s_{v} \sigma}{4 \rho_{v}}$.

- $\mathrm{R}_{0}$ and $\mathrm{u}_{0}$ are computed using $R_{0}=\frac{e}{\sigma} \frac{R_{00}}{1+a m} ; u_{0}=u_{00} \frac{\sigma}{\tau}$ 
with $R_{00}=0.05 \mathrm{~kg} \cdot \mathrm{m}^{-2} \cdot \mathrm{s}^{-1}, u_{00}=80 \mathrm{~kg}^{-1} \cdot \mathrm{m}^{3}$. Nevertheless, it will be possible to fit these last two parameters around values given by (15) for higher precision.

\section{3- Model validation}

\section{1 - Comparison with semi-empirical models}

We recall that our simplified physical model is based on an algebraic relation: $R=$ $R(U, \alpha, m, f)$, as supported by semi-empirical models, and especially by the Rothermel model. Each one leads to short computation times. The present model presents an a priori larger range of validity than semi-empirical ones: it is based on a simplified formulation of the conservation laws, and only requires two fire front positions for its set-up, whereas several are needed for semi-empirical models. Finally, the following example illustrates that the accuracy of our model is greater than that of semi-empirical models.

Weise and Biging [26] performed a set of lab scale fire experiments, for different wind and slope conditions. Then, they measured the maximum error between the experimental results and the ones provided by semi-empirical models, as reported in Table 1.

The first three models presented some significant departures from the experimental results, with Rothermel-Albini's model offering the best accuracy.

Figure 5 presents the values provided by the experiments and the Rothermel-Albini model from [26], along with those given by the present simplified physical model, for the most discriminating case, a slope angle of $30 \%$. It can be observed that the maximum error for the present model is less than $10 \%$.

\section{2 - Comparison with different experiments (laboratory and field scale)}

Some of the following cases were already tested with the previous version of this model [1]; the great advantage of the new approach is that the main part of the parameters is a priori 
computed and no longer fitted. This allows taking into account the dependence of these parameters to the physical characteristics of the vegetation. The first nine cases are lab scale (pine needle bed) or 'tunnel scale' fires, whereas the last two ones are field scale fires. The numerical values of the model parameters are given in Table 2.

Case 1 - Mendes-Lopes et al. [19] performed a series of fine scale experiments with beds of pine needles (Pinus Pinaster- PP) under several sets of slope and wind conditions in a fire tunnel.

Here, the three parameters are a priori computed using Eq. (14) and Eq. (15); one obtained different values for $\mathrm{R}$ as a function of the slope and wind conditions. These are reported on a single master graph for the sake of clarity, giving R versus $\gamma$ (Fig. 6).

Case 2 - In the same study, Mendes-Lopes et al. re-performed the same experiment, increasing the moisture content in the vegetal with $\mathrm{m}=18$. The model involves the moisture content acting through $\mathrm{A}$ and $\mathrm{R}_{0}$, which were therefore computed again using Eq. (14) and Eq. (15). $\mathrm{u}_{0}$ is not affected by the parameter $m$, so it remained equal to its previous value. With these three parameters a priori computed, it can be observed that the agreement of the curve $R(\gamma)$ with the experiments is very good (Fig. 6).

Case 3 - Mendes-Lopes et al. [20] also performed some experiments using Pinus Halepensis (PH). The parameter computation was performed using Eq. (14) and Eq. (15) and the values are reported in Table 2. It is important to note that $\mathrm{u}_{0 \mathrm{PH}}$ was computed from $\mathrm{u}_{0 \mathrm{pp}}$ with $\mathrm{u}_{0 \mathrm{PH}}=$ $\mathrm{u}_{0 \mathrm{PP}} \sigma_{\mathrm{PH}} \tau_{\mathrm{PP}} / \sigma_{\mathrm{PP}} \tau_{\mathrm{PH}}$. It can be observed that PH does not fully burn, as PP does: $\sigma_{\mathrm{ph}} / \sigma_{\mathrm{pp}}=0.85$; this lower burning rate is due to the fact that part of the vegetal stratum is lower than the optical depth $\delta$. It is therefore heated less and fuel consumption ratio is lower. 
When comparing the experiments with the two species Pinus Pinaster (PP) and Pinus Halenpesis $(\mathrm{PH})$, it can be observed that the curves $\mathrm{R}_{\mathrm{PP}}(\mathrm{U})$ and $\mathrm{R}_{\mathrm{PH}}(\mathrm{U})$ (for $\alpha=0$ ) collapse into a single master curve, despite the very different parameters:

$$
\mathrm{A}_{\mathrm{PP}}=1.25 ; \quad \mathrm{A}_{\mathrm{PH}}=1.5 ; \quad \mathrm{u}_{0 \mathrm{PP}}=2 \mathrm{~m} \cdot \mathrm{s}^{-1} ; \mathrm{u}_{0 \mathrm{PH}}=1.35 \mathrm{~m} \cdot \mathrm{s}^{-1} .
$$

When changing from $\mathrm{PP}$ to $\mathrm{PH}$, the parameter $\mathrm{A}$ increases, causing $\mathrm{r}$ to also increase. At the same time, the parameter $\mathrm{u}_{0}$ decreases, leading $\gamma$ to increase. At the end, these two opposite effects compensate for one another, as observed in Figure 5. In contrast, $\mathrm{R}_{0}$ decreases by half. Mendes-Lopez also observed that the average flame height in the $\mathrm{PH}$ experiments was half that of the PP experiments. This is also shown by the model, because, by using $H=u^{2} / g^{*}$ (4), we have $\frac{H_{P H}}{H_{P P}}=\left(\frac{u_{P H}}{u_{P P}}\right)^{2}=0.56$.

Case 4 - Viegas et al. [21] gave the variation of $r$ as a function of wind velocity, for a layer of Pinus Pinaster. By the use of these calculated parameters, we obtained a very good agreement between our model and their experiments (Fig.7).

Case 5 - Guijarro et al. [22] also used needle layers of Pinus Pinaster, and measured $r$ with two different loads. It can be seen (Fig. 8) that the values of $r$ are quite equal for both load because the higher fuel load induces greater values of both $\mathrm{A}$ (increasing $\mathrm{r}$ ) and $\mathrm{u}_{0}$ (decreasing r).

Case 6 - Porterie et al. [23] used a layer of pine needles from Pinus Pinaster. Our model prediction was in good agreement with the one provided by the small network calculation that they used (Fig. 9). 
The variation in the experimental results from the different researchers for layers of pine needles is due to secondary factors: ambient temperature, relative moisture content of the air, display of the layer and width of the fire front. This shows that in the case of wildfires, a high accuracy cannot be reached with models and that only the general tendencies may have significance.

We observe that $R_{0}$ is variable and the model cannot always predict it exactly. On the other hand, it is the easiest parameter to fit for a fire, taking the value for a null slope and no wind.

Case 7 - Anderson and Rothermel [24] studied the effects of wind on the ROS for several moisture content values in two pine species: the Ponderosa Pine (POP) and the White Pine (WP). For each case, $A$ was calculated and $\mathrm{R}_{0}$ and $\mathrm{u}_{0}$ for the initial moisture content were fitted, because we did not know the exact values of $\sigma$ and $\tau$. They were computed for the others using the relations Eq. (14) and Eq. (15). It can be noted that $u_{\mathrm{pop}}>\mathrm{u}_{\mathrm{wp}}$ : this proceeds from the fact that the effective load for cases with POP is greater than the effective load for cases with WP (see [24]), because the optical depth for POP is greater than that for WP (Figs. 10 and 11$)$.

Case 8 - Nelson [25] considered a fuel bed of Pinus Elliottii (PE) and, in a second experiment, a bed of PE Pine was used with an additional layer of saw palmetto. This situation was more complex because the vegetal stratum was not homogeneous. However, it was the needle layer that played the main role. Once again, $u_{0}$ should have been larger because the fuel load was stronger, but the optical depth defined the useful charge. In both cases, there was an agreement between the experiment and theory, as seen in Fig. 12 a-b. 
Case 9 - The series of experiments from Weise [26] were particularly interesting because a large set of configurations was investigated: upslope or downslope fires with aiding or opposite wind flows, for several slope and wind velocity conditions.

The vegetal stratum was heterogeneous because it accounted for two components differentiated by nature and geometry. However, we considered it to be a homogeneous stratum, where every cell fully burned (according to assumption H6).

Despite the large variability of the configurations, the results presented in Fig. 13 are in very good agreement with the experimental data. There was just one experimental point $(\alpha=30 \%$; $\mathrm{U}=-0.42 \mathrm{~m} \cdot \mathrm{s}^{-1}$ ) that did not coincide with the numerical results from the modelling. Nevertheless, the authors indicated that for this point, only one experiment was performed and, therefore, the corresponding point does not coincide with the averaged results, like in the others cases. For this reason, the measurement uncertainties are important for this point.

Case 10 - Cheney et al. [27] performed some experiments with large grassland fires over several years in Australia. Assuming that each vegetal stratum was reasonably similar, we applied our models. Of course, the dispersion of the results was greater than in lab scale experiments because the comparison concerned 20 different fires. Nevertheless, the trend provided by the model was in agreement with the experiments (Fig. 14), in contrast to others modelling approaches, such as Behave, MK5, Firetec and Firestar (Morvan et al. [28]), which do not seem to be able to reproduce fire spreads under strong winds.

Case 11 - MacArthur [29] performed more than 15 grass fire experiments in different places. The result from our modelling strategy was in good agreement with the results of these experiments (Fig. 15). 
Case 12 - A global comparison between the model and experiments: the existence of a universal relation, Eq. (13), makes it possible to collapse all of the results with the same A parameter onto a single master curve. We present this collapse for the value $\mathrm{A}=1.5$, which is the most frequent case, by plotting the reduced ROS $r$ as a function of the tilt angle given by Eq. (2). We added the case studied by Catchpole [30]. The universal relation for the ROS is in very good agreement with the main portion of the experimental results, with the exception of some points for which comments were already made (Fig. 16). We also present all of the results related to natural fires at the lab scale (Fig. 17).

\section{3 - Implementation of the fire model in a simulation framework for wildfire}

To be operational, the proposed model had to be integrated in a simulator that would perform the numerical integration of the front advance. The choice of the numerical method was a key point, as the objectives of the model were clearly speed and solution robustness. We also had to provide a numerical method that could be adapted to the numerical manipulations of the underlying model.

We propose a new approach for the simulation of spatial phenomena, combining asynchronous [31] discrete event simulation [32] and front tracking [33] [34].

Front tracking methods are used to study interface dynamics, such as the frontline of a fire. By focusing on the interface, it is possible to give a simplified view of these systems.

An asynchronous front tracking method is well adapted because time is not defined as discrete. If discrete time stepping is used, the resolution of the system falls under the same limitations as with regular meshes; trade-offs have to be made between the temporal resolution and temporal scale of the simulation (Courant condition). Describing front models as discrete event systems permits us to define time as a real value, like the other dimensions of the system, rather than have to rely on a fixed time step. 


\section{Modelling description}

The method proposed in this paper was derived from the markers method, which has been proven to be relatively quick and accurate [33]. Details of the algorithms are available in [34]. The interface is discretised by a set of points or markers; markers are moved according to the rate of spread model in the direction of the front normal at this point (with a displacement vector).

The originality of the proposed front tracking markers method involves the asynchronous movement of markers, with no global time step, by using Discrete Event Simulation (DES).

In DES, the local state change of a system is triggered by an event. Each event has an occurrence time, which does not have to be spatially uniform. During s simulation, events with occurrence times are created, scheduled in an event list sorted by their occurrence times and processed asynchronously, with the most imminent event processed first. By using DES, it is possible to move every marker independently, calculating the marker advance only when it has covered a significant distance.

There are two key concepts that form the basis of the simulation, since they generate the driving events:

- The quantum distance defines (in meters) the distance to be covered by a marker advance. The actual resolution of the simulation is limited by this quantum distance and details that are smaller than this quantum distance may not be taken into account. The time it takes for a marker to travel the quantum distance will define the time to the next event. By this means, a fast marker will generate more events and calculations than a small marker.

- A collision occurs when a marker is moving into a different area (from bushes to a fuel break, or from an un-burnt area to an already burnt area). Collisions also occur if 
a marker and its neighbour marker are separated by less than the quantum distance. Each collision triggers a dynamic modification of the shape, by adding or deleting markers and merging firelines.

All motion and all events are generated either by a collision or by a marker planning an advance in space and time. Collisions trigger the resolution of the overall shape modification, while markers actually move the quantum distance by self-activation until they stop.

Marker advance is integrated by a simple Euler approximation, with the marker direction and speed given by the fire propagation model. Local slope angle and wind speed for the propagation model at the very location of a marker is approximated by bi-cubic interpolation if the elevation and wind data are in the grid format.

\section{Lançon benchmark results}

Since the goal of the method was to be able to simulate a real fire, a simulation based on a real scenario is presented to verify the robustness and speed of the method in propagating $2 \mathrm{D}$ fire fronts. To do this, we selected the '2005 Lançon' fire, which took place in the south of France and burned about $800 \mathrm{Ha}$ of shrubs and forest. On that day, a North Westerly wind of $50 \mathrm{Kmh}^{-1}$ was blowing, providing extreme propagation conditions. The simulation was crossvalidated with the FARSITE simulator [13]. This fire area simulator is used extensively by the USDA and is considered to be a reference in forest fire simulation. The simulation time with this tool on a desktop PC is on the order of 10 minutes for a large wildfire.

This accident was well documented, with road network, ignition points and three contours of the fire front over time.

The simulation was run with a quantum distance of 3 meters. An orographic wind map was calculated as a stationary solution using mainstream computational fluid dynamics software (Fluent). The resolution of the wind map was the same as the digital elevation model: $50 \mathrm{~m} \times$ 
$50 \mathrm{~m}$. In the Farsite simulation, the spatial resolution was set as 20 meters, with a temporal resolution of 5 minutes.

The rate of spread model used a set of parameters that corresponded to a brush fire fitted by linear regression using the observations of the first contour (time 12:00) and ignition points (time 09:40) with the method described in [1]. The 'brush' fuel model was selected in Farsite for this simulation, with adjustments made so that the first simulated contour also matched the first observed contour.

The simulation results and observations are presented in Fig. 16. The simulation was started at time 9:40 at the exact same points as the estimated ignition points, and stopped at time 16:30. While every detail larger than three meters was taken into account, the simulation time using this quantum distance was under a minute on a $2.5 \mathrm{GHz}$ single core processor with $1 \mathrm{~GB}$ of RAM. The Farsite simulation duration for the same case was 12 minutes, and using the smaller spatial and temporal resolution of Farsite resulted in the simulated fire line crossing the motorway, which is not presented here. Figs. 16, 17 and 18 present the simulation results for the proposed model and Farsite. It can be observed that the Farsite simulation showed strong expansions to the side, which did not occur in the real fire. Nevertheless, both simulations are in good accordance with the observed front. Even if both simulators are considered to have achieved good results, there are two main advantages of the proposed model. The simulation time was significantly lower on the proposed model and there was no need to find the right fuel model (as parameters are fitted using the first available contour) in order to perform a realistic simulation of the front line behaviour. These advantages can be important when the fuel type is relatively unknown and many fire fighting scenarios need to be tested in a short time.

\section{5 - Conclusion}


We proposed a model that presents the following properties:

- It obeys the physical laws of the phenomenon, leading it to be a priori usable in every configuration.

- However, these laws are simplified using ten main assumptions to allow its reduction to a single algebraic equation: one that gives the ROS as a function of wind, slope and vegetation. The computational time is therefore quite negligible.

- This model is full-physical and 3D: this leads to a complete set of geometrical variables (height, depth and tilt angle) and thermodynamic quantities (temperature, radiant flux and fire front intensity).

- There are only three parameters for characterizing the vegetal stratum; they are calculated using physical data for the vegetal fuel.

- It exhibited very good agreement with a large set of lab scale experiments and two field scale experiments.

- Its implementation into an efficient simulator is straightforward and easy to use, leading to the accurate prediction of a real wildfire.

In the next papers, we will present different versions and specific applications of this model.

Acknowledgements: The authors are very grateful to the French Research Group 'Incendies' for the availability of the benchmark Lançon, as well as for their production of the orographic and wind map. 


\section{References}

[1] J.H. Balbi, J.L. Rossi, T. Marcelli and P.A. Santoni, Comb. Sci. Tech. 178 (2007) 2511-

2537. [2] P. Fernandes, Forest Ecol. and Man. 144 (2001) 67-74.

[3] N.P. Cheney, J.S. Gould, N.R. Catchpole, Int. J. Wildland Fire 8 (1998) 1-13.

[4] R.C. Rothermel, A mathematical model for predicting fire spread in wildland fuels, INT-

115, USDA, Forest Service Research, 1972.

[5] P.J. Pagni, T.G. Peterson, Proc. Combust. Inst., 1973, 1099-1107.

[6] F. Morandini, P.A. Santoni, J.H.Balbi, Fire Safety J. 36 (2001) 519-543.

[7] R.O. Weber, Combust. Flame 78 (1989) 398-408.

[8] O. Sero-Guillaume, S. Ramezani, J. Margerit, D. Calogine, Int. J. Therm. Sci 47 (2008) 680-694.

[9] A.M. Grishin, Mathematical modeling of forest fires and new methods of fighting them, Publishing house of the Tomsk state university, Albini, 1997.

[10] M. Larini, F. Giroud, B. Porterie, J.C. Loraud, Int. J. Heat Mass Trans. 41 (1998) 881897.

[11] R.R. Linn, J. Reisner, T. Coleman, J. Winterkamp, Int. J. Wildland Fire 11 (2002) 1-14.

[12] P.L. Andrews, Behave: fire behaviour prediction system, BURN-subsystems, Part: 1, USDA, Forest Service Research, 1986.

[13] M.A. Finney, Farsite: user's guide and technical documentation, RMRS-RP-4, USDA, Forest Service Research, 1998.

[14] B.J. McCaffrey, Purely buoyant diffusion flame: some experimental results, NBSIR 79$1910,1979$.

[15] X. Silvani, F. Morandini, Fire Safety J. 44 (2009) 279-285.

[16] J. Lozano, W. Tachajapong, H. Pan, A. Swanson, C. Kelley, M. Princevac, S. Mahalingam, Experimental Investigation of the Velocity Field in a Controlled Wind-aided 
Propagating Fire Using Particle Image Velocimetry, Ninth International Symposium on Fire Safety Science, Karlsruhe, 2008.

[17] V. Tihay, A. Simeoni, P.A. Santoni, L. Rossi, J.P. Garo, J.P. Vantelon, Int. J. Therm. Sci. 48 (2009) 488-501.

[18] B.J. McCaffrey, Some measurements of the radiative power output of diffusion flame. WSS/CI81-15. Western States Meeting of the Combustion Institute. Pullmann, Washington, 1981.

[19] J.M.C. Mendes-Lopes, J.M. Ventura and J.M. Amaral Int. J. of Wildland Fire 12 (2003) $67-73$.

[20] J.M.C. Mendes-Lopes, J.M.P. Ventura, For. Ecol. and Man., 234, Suppl. 1, 2006, 116.

[21] D.X. Viegas, Int. J. Wildland Fire 13 (2004) 101-117.

[22] M. Guijarro C. Hernando, J.A. de los Santos, C. Diez, Forest fire behaviour in the wind tunnel, Hephaistos Project Report, ENV4-CT96-0299, 1997.

[23] B. Porterie, N. Zekri, J.P. Clerc and J.C. Loraud, Comb. Flame 149 (2007) 63-78.

[24] H.F. Anderson and R. Rothermel, Proc. Comb. Inst., 1965, 1009-1019.

[25] R.M. Nelson and C.W Adkins. Can. J. For. Res. 16 (1986) 1293-1300.

[26] D. Weise and G. Biging, Forest Science, 43 (1997) 170-180.

[27] N.P. Cheney and J.S. Gould, Int. J. Wildland Fire, 5 (1995) 237-247.

[28] D. Morvan, J.L. Dupuy, F. Pimont, R.R. Linn, For. Ecol. Man. 15 (2006) 90.

[29] A.G. MacArthur, Mass Fire Symposium, Canberra, Australia, 1969.

[30] W.R. Catchpole, E.A. Catchpole, B.W. Butler, R.C. Rothermel, G.A. Morris and D.J. Latham. Comb. Sci. Tech. 131 (1998) 1-37.

[31] H. Karimabadi, J. Driscoll, Y.A. Omelchenko, N. Omidi, J. Comp. Phys. 205 (2005) 755-775

[32] B. Zeigler, Theory of Modeling and Simulation. 2nd Edition, Academic Press, 2000. 
[33] N. Risebro, H. Holden, Front Tracking for Hyperbolic Conservation Laws, Springer, Berlin, 2002.

[34] J.B. Filippi, P.A. Bisgambiglia, A DEVS Front tracking method applied to forest fire simulation, in: Proc. of the SCS 2007 conference, IEEE/AIS, Issues 1, Buenos Aires, Argentina 2007.

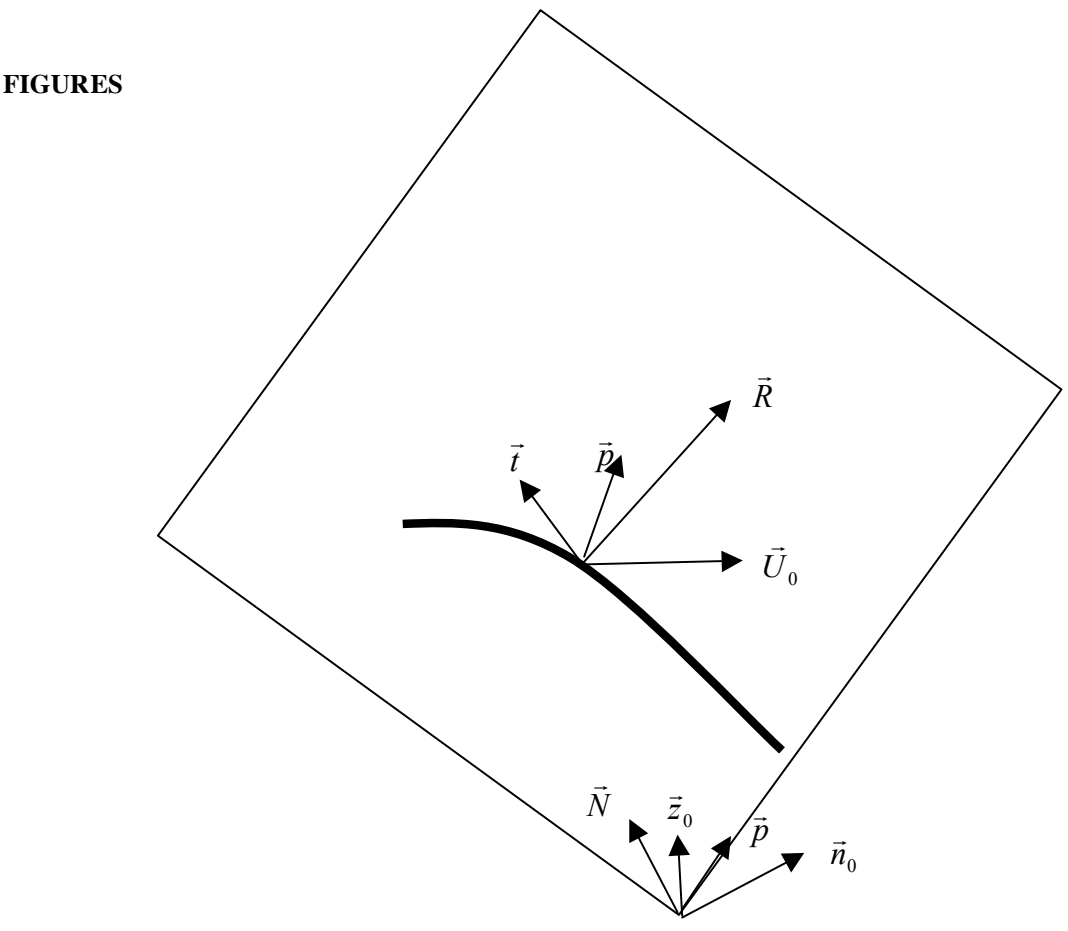

Figure 1 : Flame front over a tilted plane, under wind aided conditions 


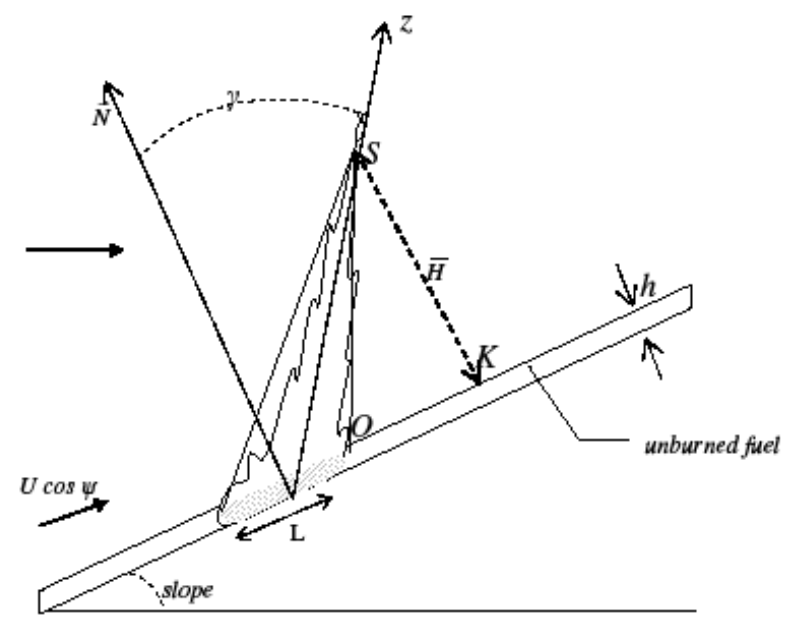

Figure 2 : Norma cut in the vertical plane of the flame front

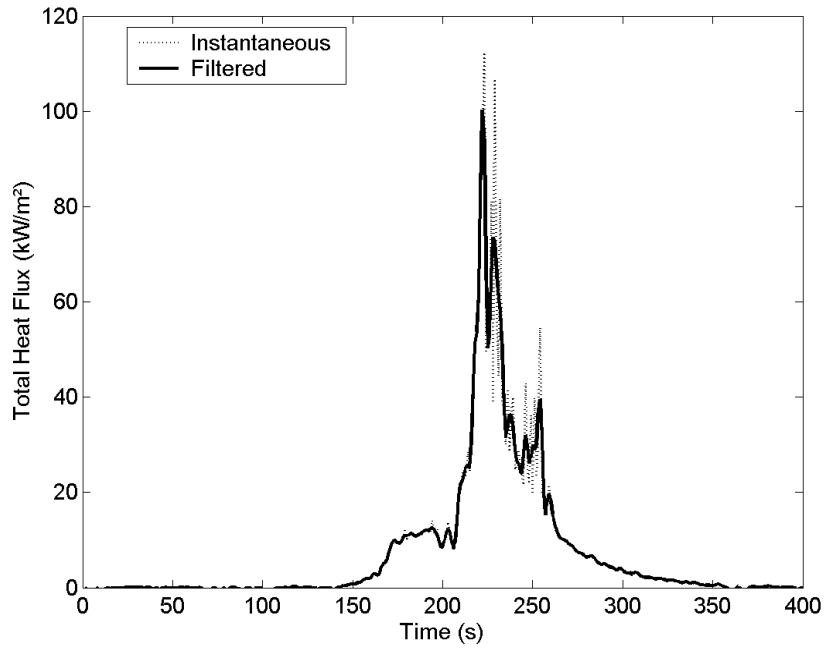

Figure 3 : Total heat flux density versus time from a field scale experiment (Courtesy Silvani and Morandini [4]) 

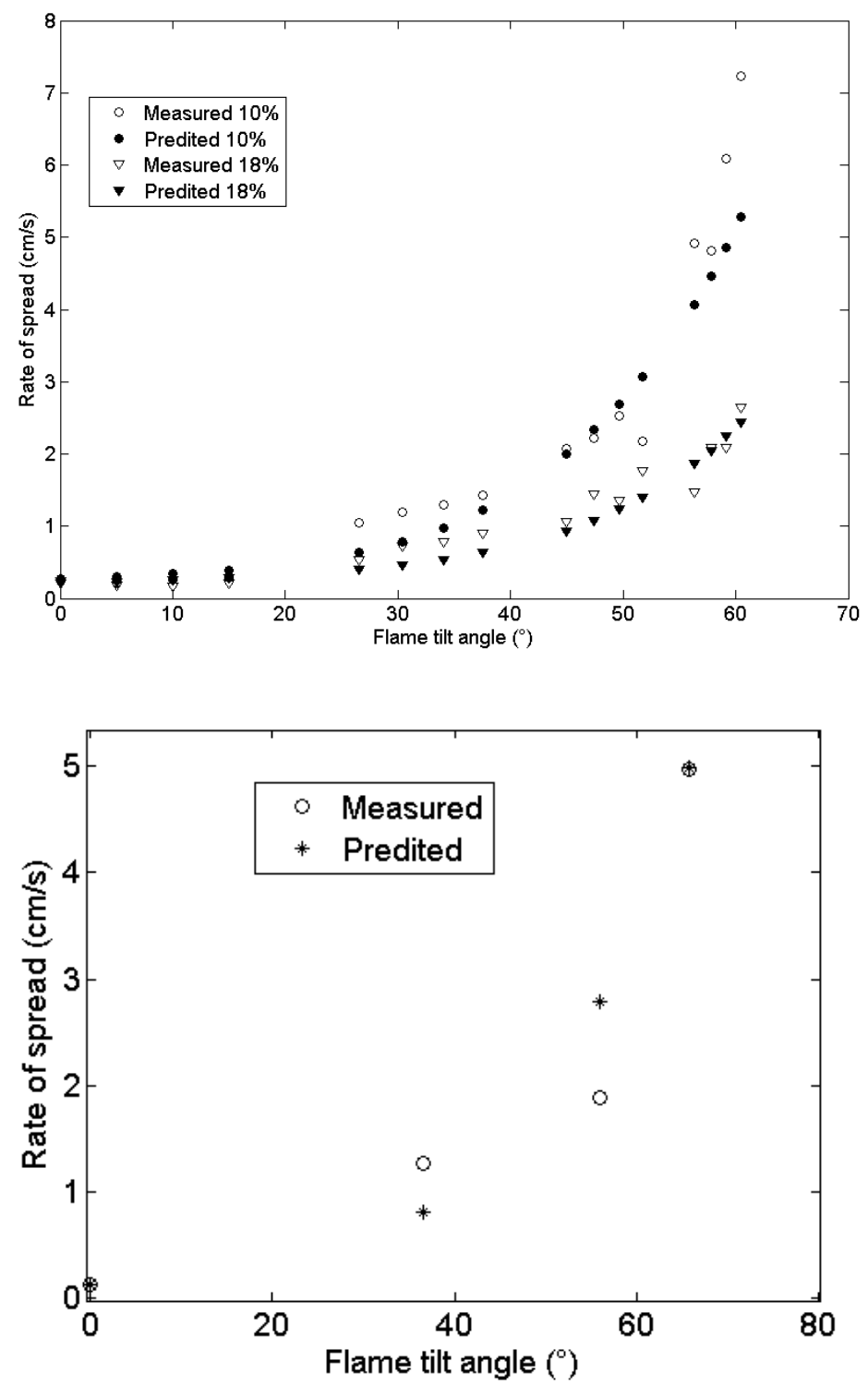

Figure 5 : Comparison model with a wind aided fire experiment of pine needles under slope conditions.

top-Pinus Pinaster in [6]; bottom- Pinus Halenpesis in [7] 


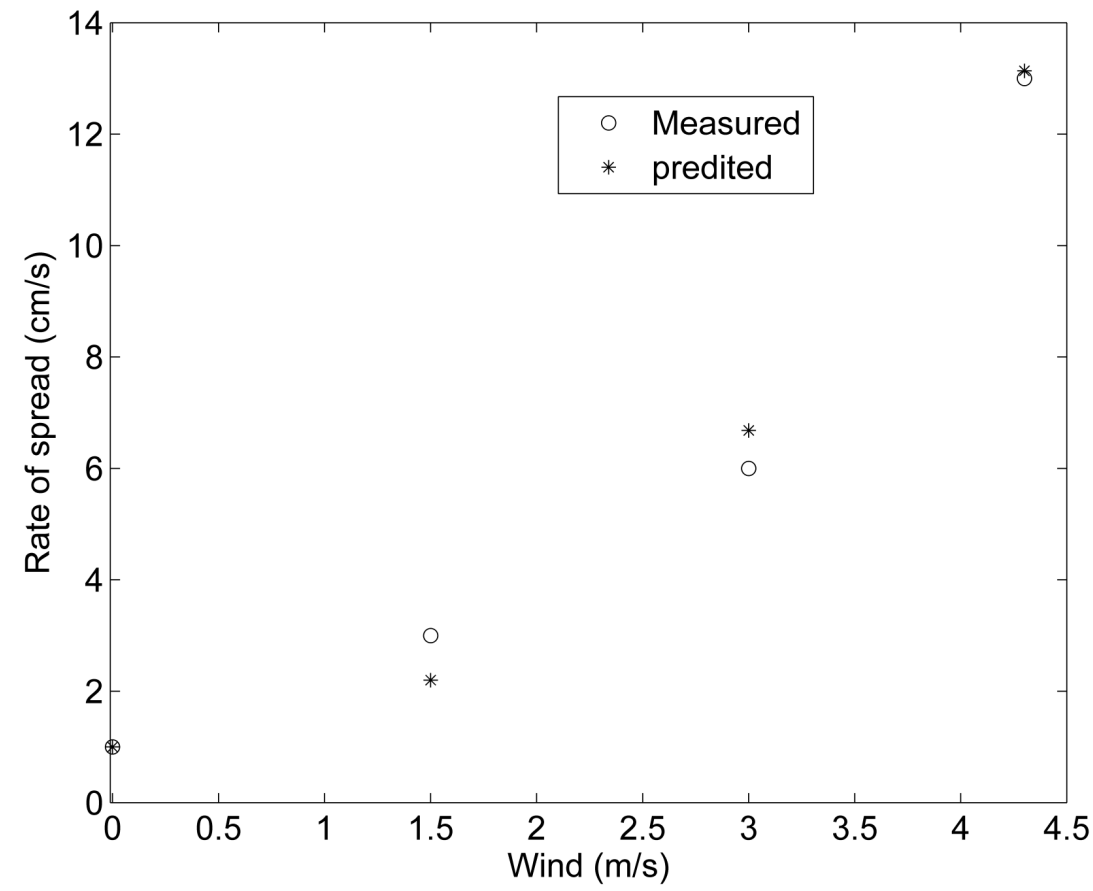

Figure 6 : Comparison model with a wind aided fire experiment of pine needles under null slope conditions 

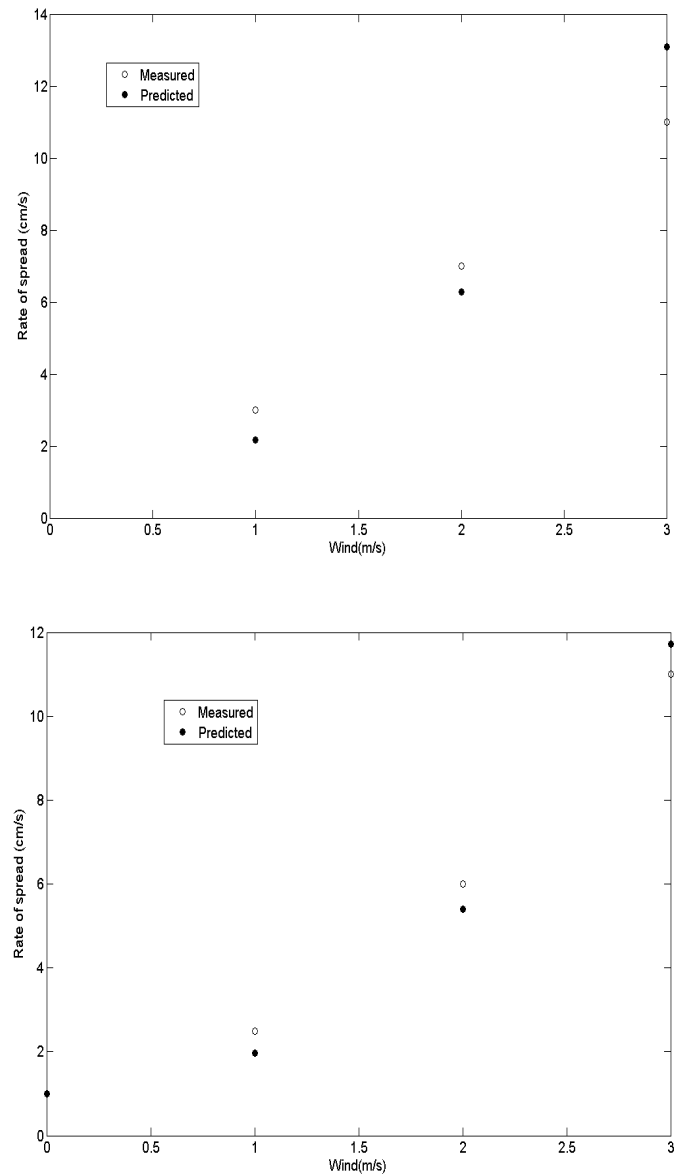

Figure 7 : Series of fire experiments with pine needeles (PP) under null slope conditions $\left(\sigma=0.5 \mathrm{~kg} \cdot \mathrm{m}^{-2}\right.$ and $\left.\sigma=1 \mathrm{~kg} \cdot \mathrm{m}^{-2}\right)$ 


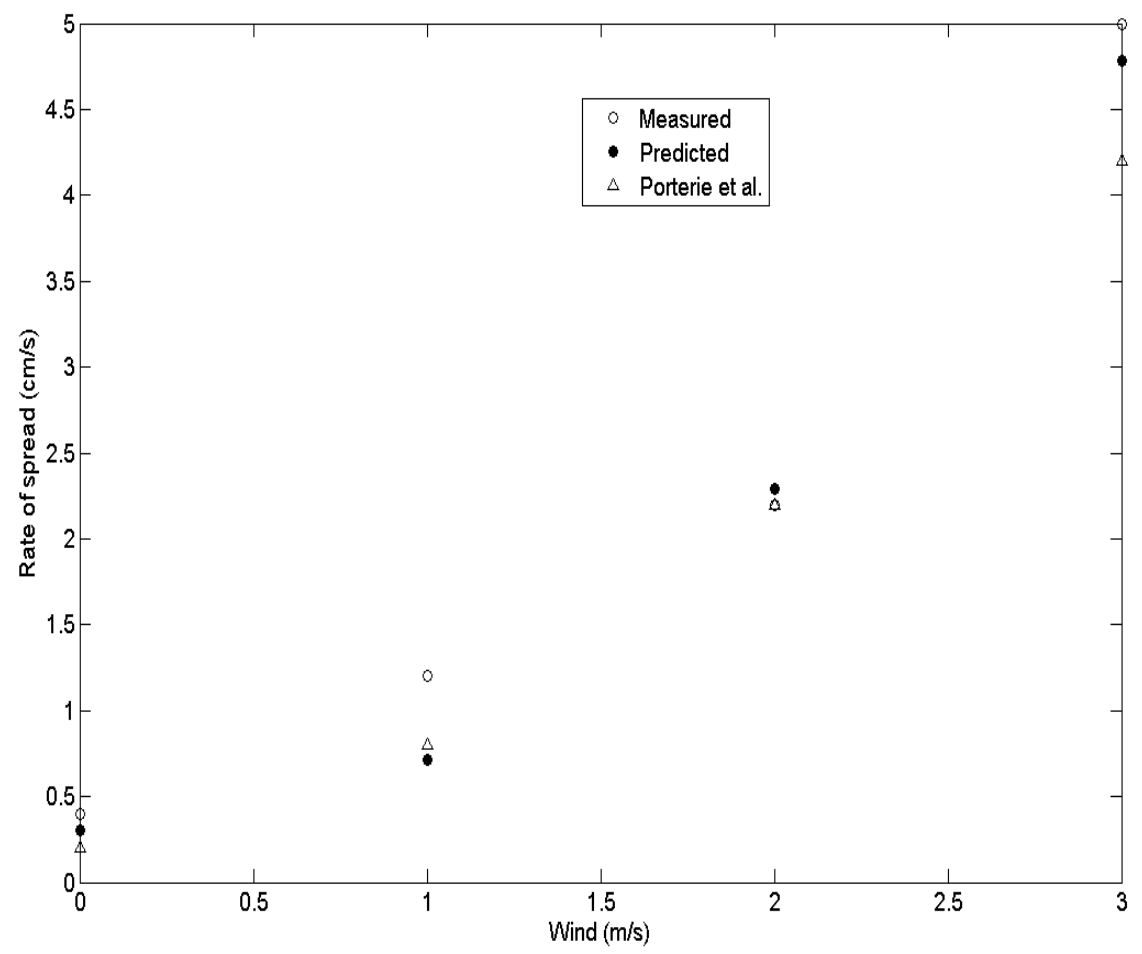

Figure 8 : Comparison of the model with wind aided fire experiments of pine needles (Ponderoso Pine $-\sigma=1 \mathrm{~kg} \cdot \mathrm{m}^{-2}$ ) under null slope conditions [10] 


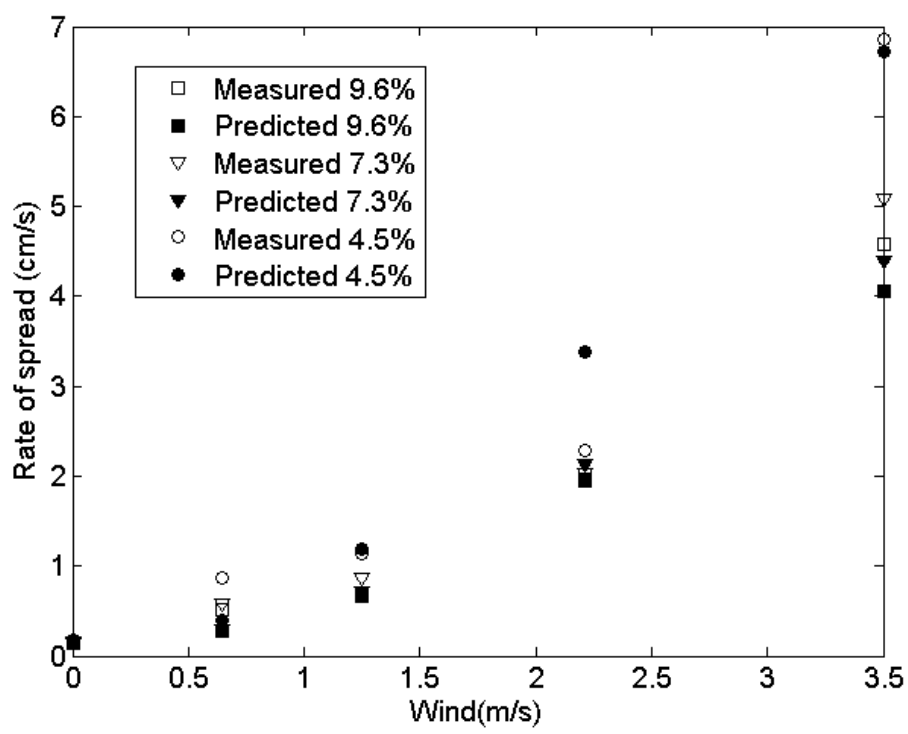

Figure 9 : Comparison model with wind aided fire experiments of pine needles (Ponderoso Pine) under null slope conditions [11]

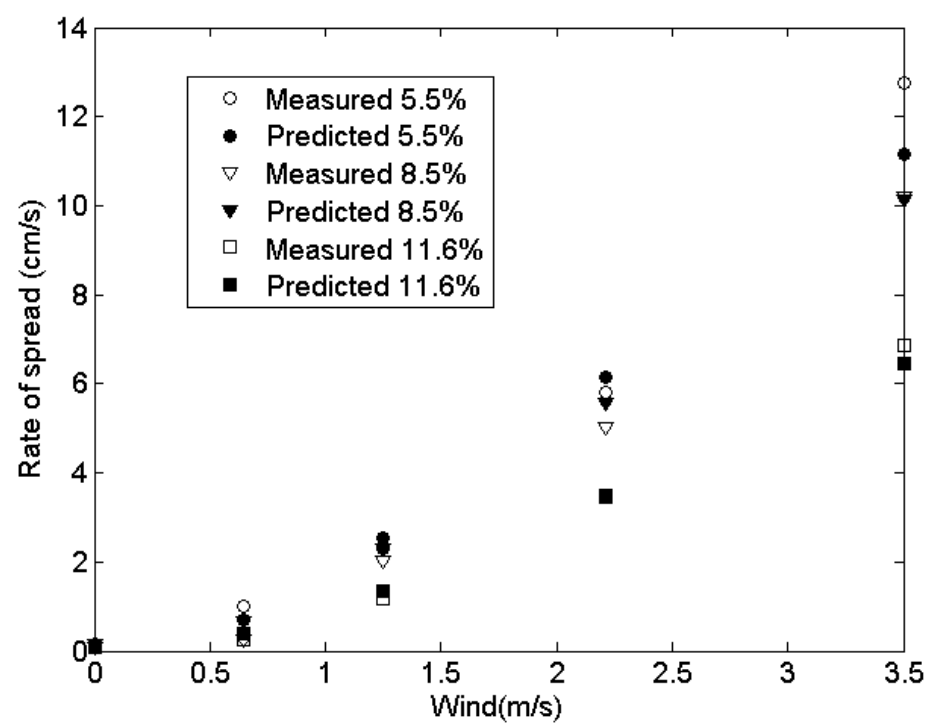

Figure 10: Similar results with White Pine 

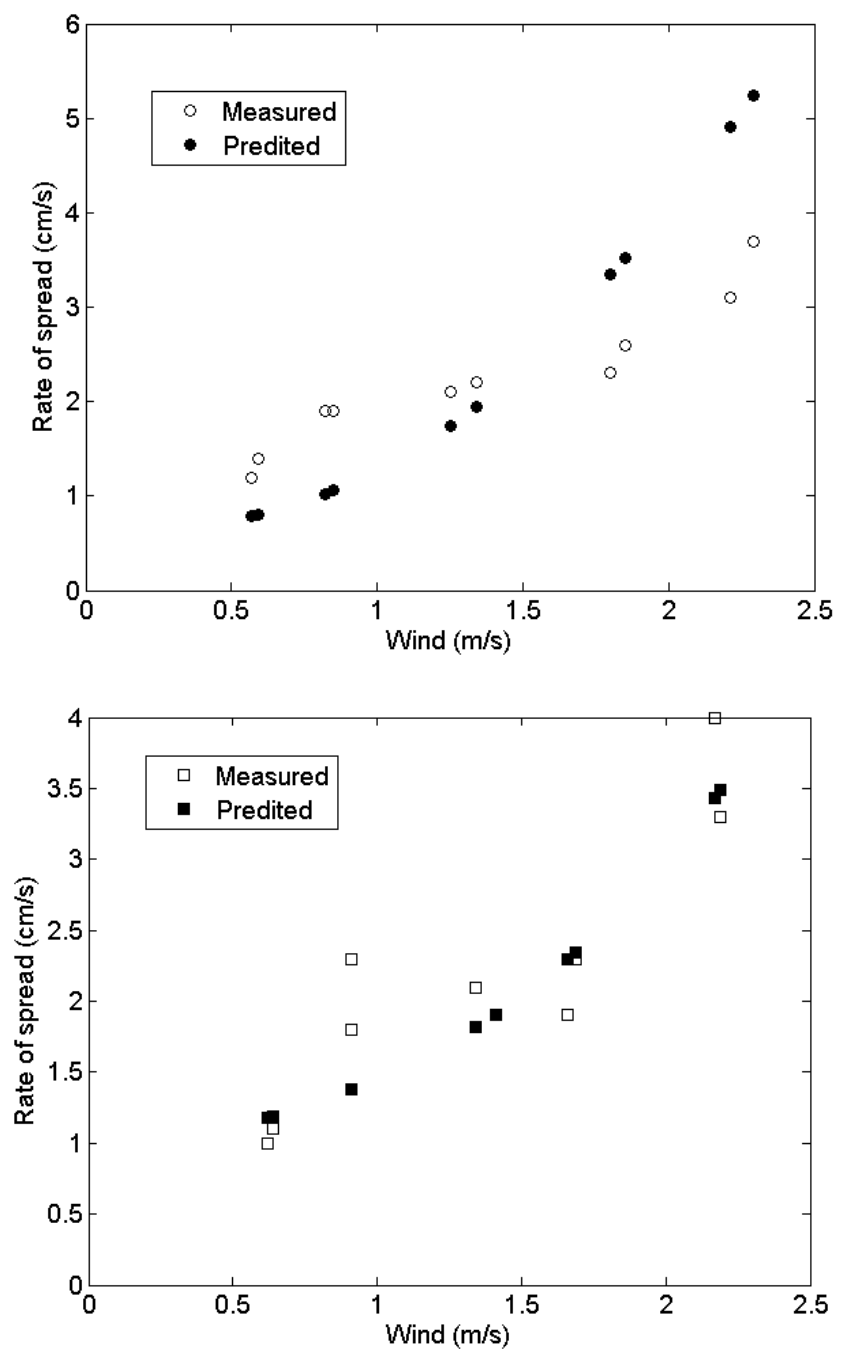

Figure 11: Comparison model with wind aided fire experiments of pine needles (top - Pinus Alliotti; bottom- Saw Palmetto ) [12] 


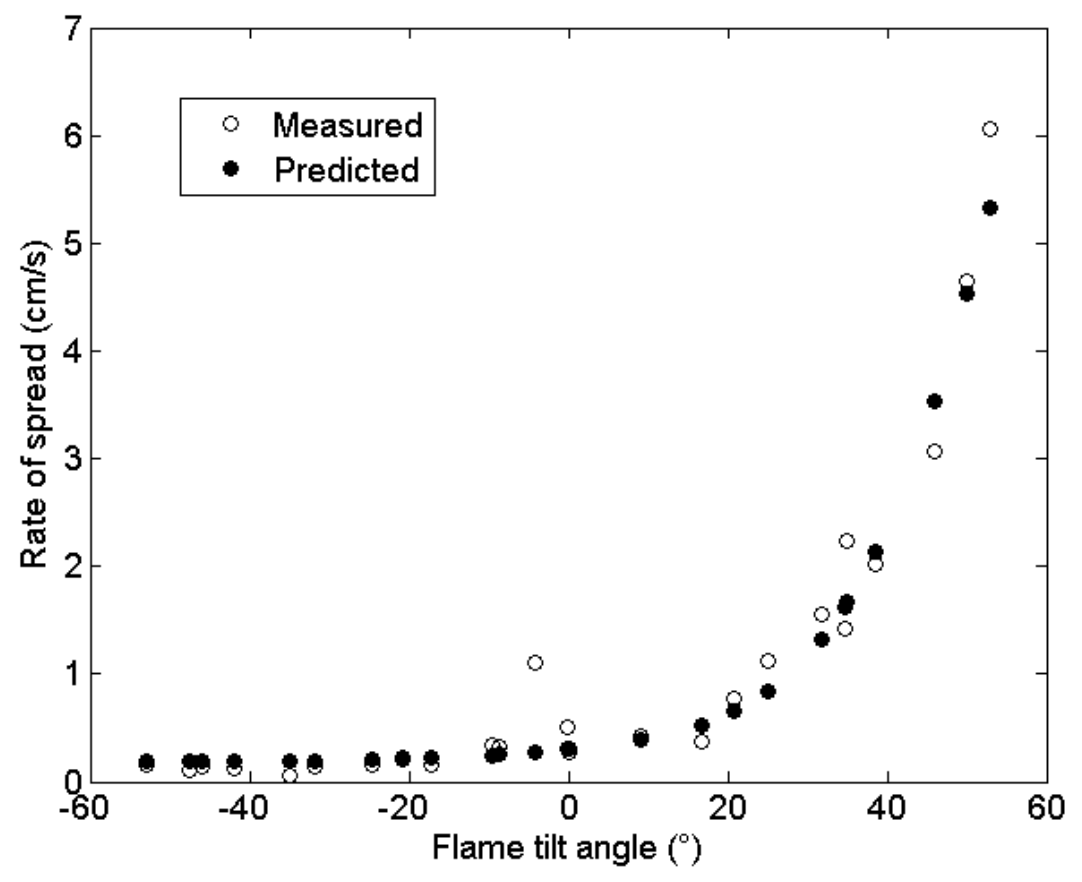

Figure 12: Comparison model with wind aided fire experiments of pine needles in a tilting tunnel for positive abd negative slope and ind velocity [13] 


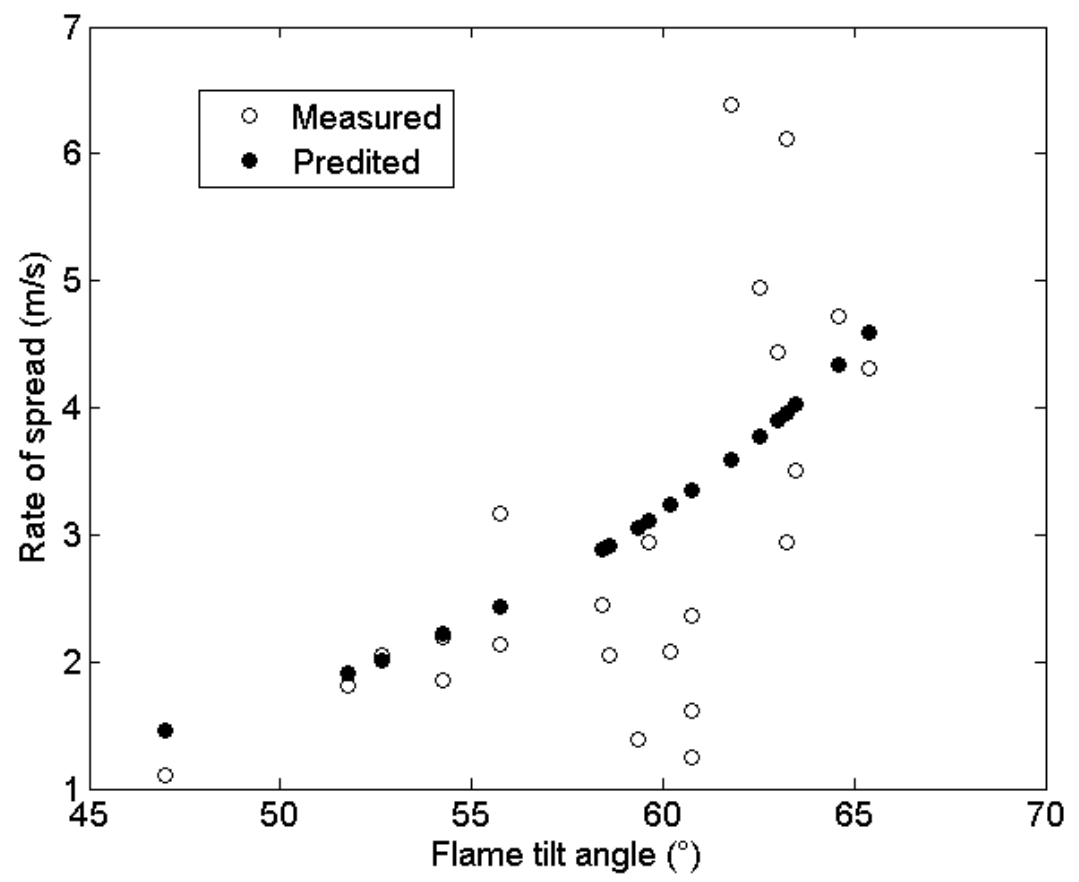

Figure 13: Comparison model with grassland fire experiments [14] 


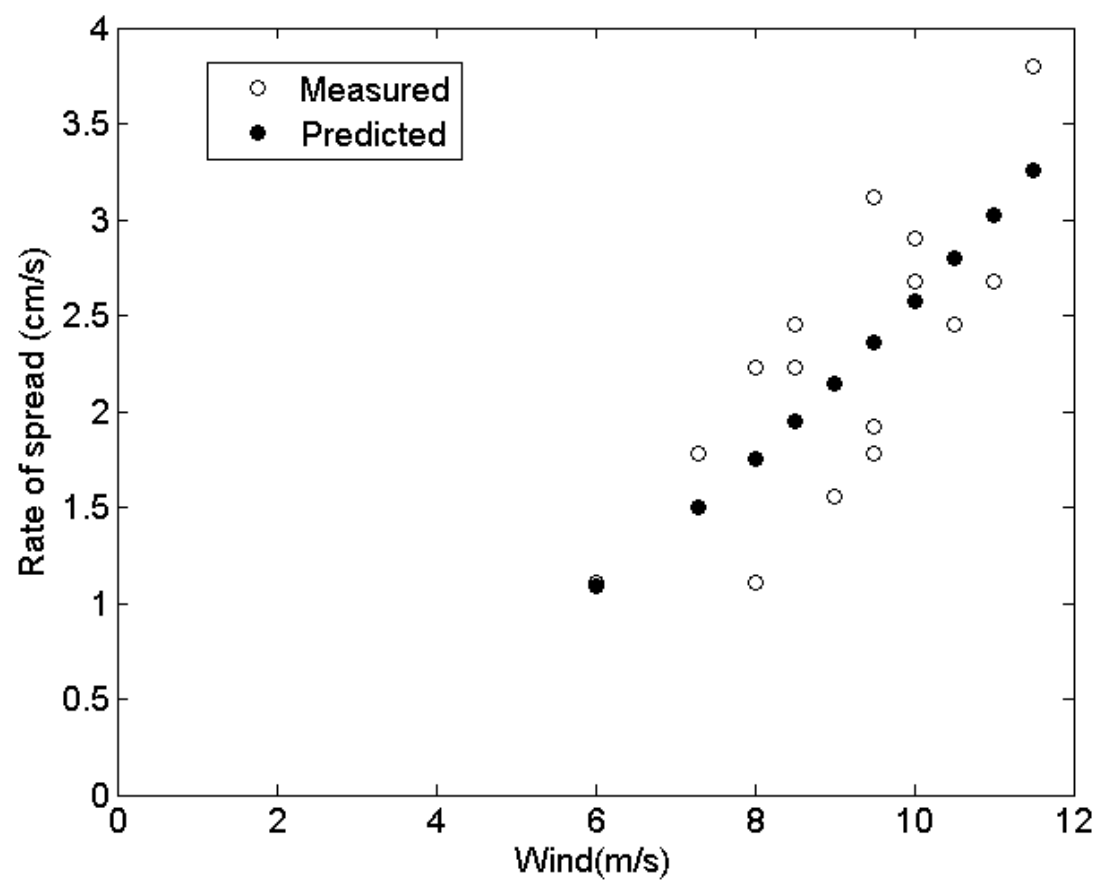

Figure 14: Grassland fires from [16]

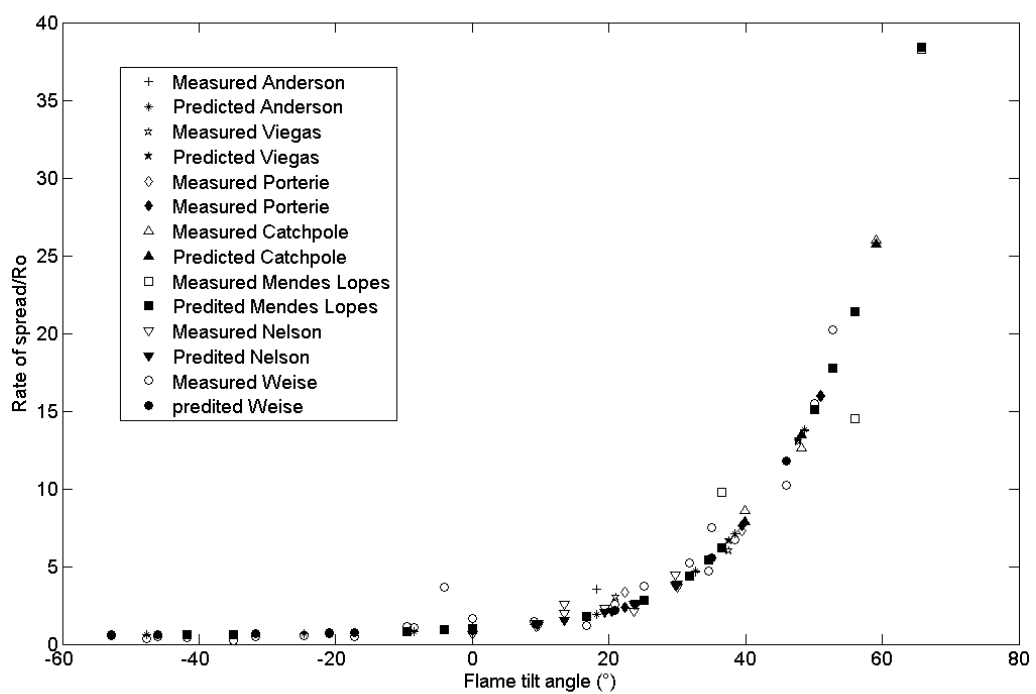

Figure 15: A synthesis of different provided comparisons between models and experiments 


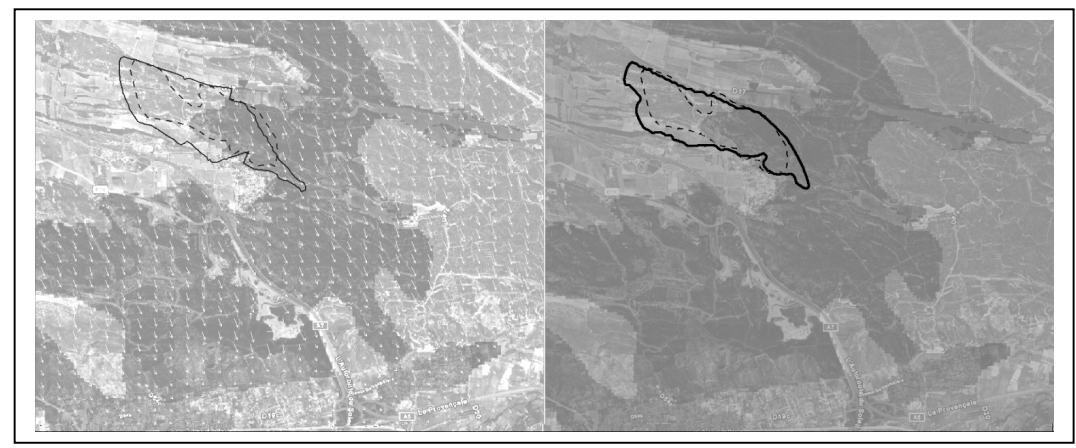

Figure 16 : Proposed (left) and Farsite (right) simulation results of the Lançcons benchmark of the fire at $12 \mathrm{~h}$. Dashed line represents the observed burned area.

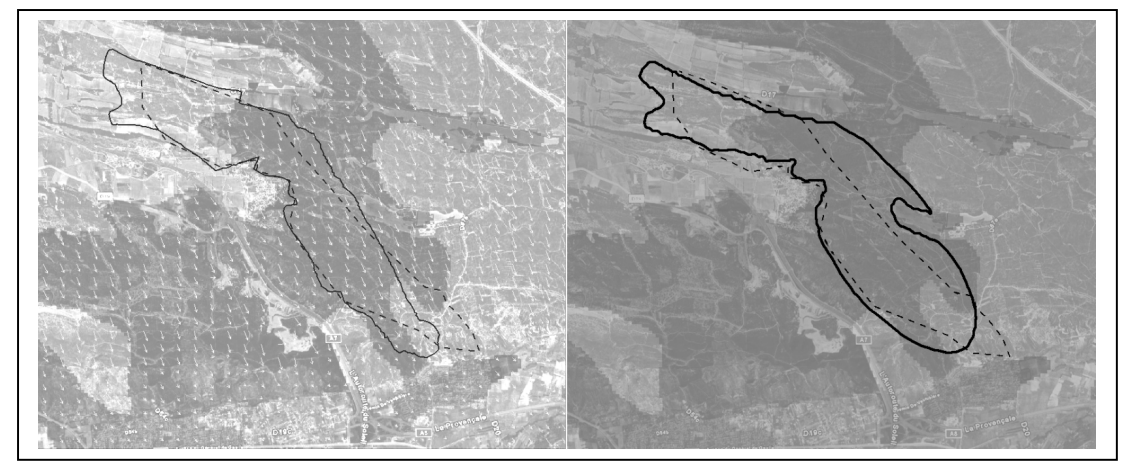

Figure 17 : Proposed (left) and Farsite (right) simulation results of the Lançcons benchmark of the fire at $14 \mathrm{~h} 30 \mathrm{~h}$. Dashed line represent the observed burned area.

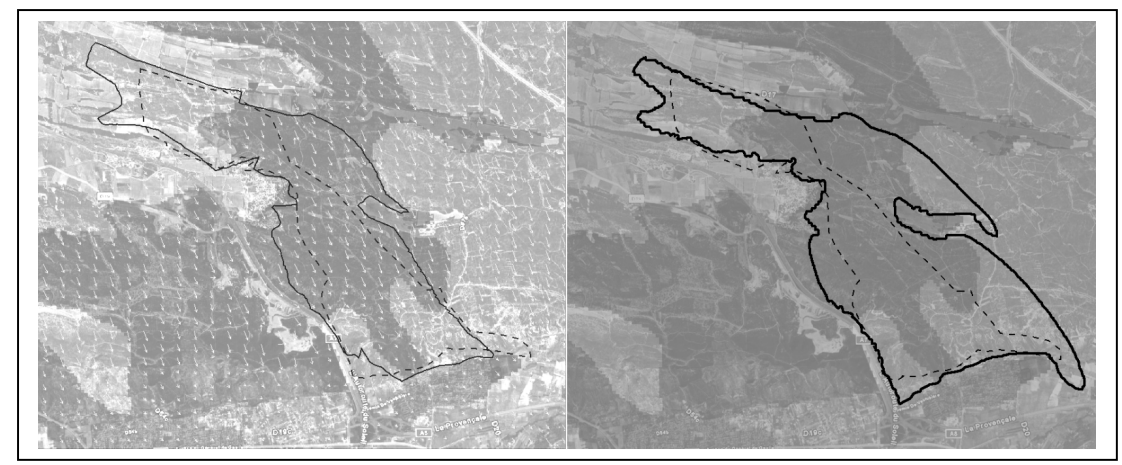

Figure 18 : Proposed (left) and Farsite (right) simulation results of the Lançcons benchmark of the fire at $16 \mathrm{~h} 30$. Dashed line represents the observed burned area. 
